\title{
A Counterexample to Uniform Approximation on Totally Real Manifolds in $\mathbb{C}^{3}$
}

\author{
ERLEND FORNÆSS WOLD
}

\section{Introduction and Main Result}

Let $X$ be a closed subset of $\mathbb{C}^{n}$. We say that $X$ admits uniform approximation if for any continuous function $f \in \mathcal{C}(X)$ and for all $\varepsilon>0$ there exists a holomorphic function $g \in \mathcal{O}\left(\mathbb{C}^{n}\right)$ such that $\sup _{x \in X}|f(x)-g(x)|<\varepsilon$. We will be concerned with the case where $X$ is a totally real manifold: a smooth manifold whose tangent space at no point contains a nontrivial complex subspace (in which case there are no Cauchy-Riemann equations on $X$ !).

One of the first results in approximation theory in complex analysis was the well-known theorem of Weierstrass [15]: If $X \subset \mathbb{C}$ is an in interval of the real line, then $X$ admits uniform approximation. This result was generalized by Carleman [4] to the effect that $X$ could be taken to be the entire real line in the complex plane. A complete characterization of the subsets of $\mathbb{C}$ that admit uniform approximation now exists: $X$ admits uniform approximation if and only if (i) $\mathbb{C} \backslash X$ has no relatively compact components, (ii) $X$ has no interior, and (iii) $\mathbb{C} \backslash X$ is locally connected at infinity (see e.g. [14]). In particular we have that a closed smooth 1-dimensional submanifold of the complex plane admits uniform approximation.

When considering the state of affairs in several complex variables it is natural to consider the compact and noncompact cases separately. Hörmander and Wermer showed that if $X$ is a polynomially convex compact totally real manifold then $X$ admits uniform approximation [7]. It is also possible to get $\mathcal{C}^{k}$-approximation on $X$-depending on the smoothness of $X$ and the function $f$ [11].

In the noncompact case the situation is best understood if the "size" of $X$ is small. Following the work of Alexander [1], Gauthier and Zeron [5] have shown that uniform approximation is possible if $X \subset \mathbb{C}^{n}$ is a locally rectifiable dendrite-that is, if it is closed and connected and has locally finite 1-dimensional measure and if $\check{H}^{1}(X, \mathbb{Z})=0$. In the case of "bigger" sets it is known that one can take $X=$ $\mathbb{R}^{n} \subset \mathbb{C}^{n}[6 ; 12]$. Manne [9] has shown that if $X$ is the union of two totally real planes such that $X$ is polynomially convex, then $X$ admits uniform approximation.

The purpose of this paper is to produce an example that demonstrates the following theorem.

THEOREM 1.1. There exists a proper $\mathcal{C}^{\infty}$-smooth embedding $\phi: \mathbb{R}^{2} \rightarrow \mathbb{C}^{3}$ such that the following statements hold for $M:=\phi\left(\mathbb{R}^{2}\right)$ :

Received October 31, 2007. Revision received December 19, 2007. 
(A) $M$ is totally real and

(B) $M$ is polynomially convex, yet

(C) $M$ does not admit uniform approximation.

That the set $M$ is polynomially convex means that $M$ has an exhaustion of polynomially convex compact sets.

We note that even though uniform approximation by entire functions fails, Manne [8] has shown that there exists a neighborhood $\Omega$ of $M$ such that uniform approximation is possible by functions holomorphic on $\Omega$. Approximation by functions on varying neighborhoods was shown by Nunemacher in [10].

\section{Sketch of the Construction}

Before plunging into the details we shall try to explain the main idea of the construction. We will choose a smoothly bounded (topological) disk $D_{0}$ contained in the unit disk in the complex plane $\left(D_{0}\right.$ will agree with the unit disk on the right half-plane). For each $j \in \mathbb{N}$ we define the shifted disk $D_{j}=D_{0}+j \cdot \sqrt{2}$. Then $\partial D_{j} \cap \partial D_{j+1}$ will consist of exactly two points and $\partial D_{j} \cap \partial D_{k}$ will be empty when $k \neq j \pm 1$. For a specific choice of functions $f_{j} \in \mathcal{O}\left(D_{j}\right) \cap \mathcal{C}^{\infty}\left(\bar{D}_{j}\right)$ and a sequence of positive real numbers $\varepsilon_{j}$, we define the sets

(a) $A_{j}:=\left\{(z, w) \in \mathbb{C}^{2} \mid z \in D_{j}, w=f_{j}(z)+t \cdot i,-\varepsilon_{j} \leq t \leq \varepsilon_{j}\right\}$,

(b) $X_{j}:=\left\{(z, w) \in \mathbb{C}^{2} \mid z \in \partial D_{j}, w=f_{j}(z)+t \cdot i,-\varepsilon_{j} \leq t \leq \varepsilon_{j}\right\}$.

Then $A_{j}$ is a family of holomorphic disks and $X_{j}$, which is a totally real annulus, is the union of their boundaries. Now the main point is to choose the domain $D_{0}$ and the functions $f_{j}$ such that (i) $X_{i} \cap X_{j}=\emptyset$ for all $i \neq j$ and (ii) for each $j \geq 1$ there is a piece $E_{j}$ of $X_{j}$ such that $M_{j}:=\overline{X_{j} \backslash E_{j}}$ is a polynomially convex disk and $E_{j} \subset A_{j-1}$. If we choose a point $q_{j} \in A_{j}$ for each $j \in \mathbb{N}$ then it is not hard to see that uniform approximation cannot be possible on the union $\tilde{M}:=$ $\bigcup_{j=0}^{\infty}\left(M_{j} \cup\left\{q_{j}\right\}\right)$; this is demonstrated in Lemma 3.3.

In Lemma 3.1 we construct a function $f \in \mathcal{O}(\triangle) \cap \mathcal{C}^{\infty}(\bar{\triangle})$ with very specific information about the values of $f$ over certain parts of $\bar{\triangle}$. We then use this $f$ to define the domain $D_{0}$ and the functions $f_{j}$, and we proceed to show that we can likewise define sets $A_{j}, M_{j}, E_{j}$ with the desired properties (Lemma 3.2). Finally, in Lemma 3.4 we show that in $\mathbb{C}^{3}$ we can find a totally real embedded $\mathbb{R}^{2}$ that is polynomially convex and contains $\tilde{M}$.

\section{The Construction}

Let $\triangle$ denote the open unit disk in $\mathbb{C}$ and let $\triangle(\sqrt{2})$ denote the open disk of radius 1 centered at the point $\sqrt{2}$. We choose two subsets of $\triangle: A:=\triangle \backslash \overline{\triangle(\sqrt{2})}$ and $B:=\triangle \cap \triangle(\sqrt{2})$. Let $\left\{z_{1}, z_{2}\right\}=\partial \triangle \cap \partial \triangle(\sqrt{2})$ (with $z_{1}$ having positive imaginary part). Note that $\partial \triangle(\sqrt{2}) \cap \triangle \cap \mathbb{R}=\{\sqrt{2}-1\}$. Let $Q$ be the interior 
of a closed (topological) disk obtained by smoothing the corners of the square $[-1,1] \times[-1,1]$ in such a way that $Q$ is invariant under reflections with respect to both axes. Choose $Q$ such that $\{-1\} \times\left[-\frac{1}{2}, \frac{1}{2}\right] \subset \partial Q$ (the precise number $\frac{1}{2}$ is irrelevant to the construction) and such that $\partial Q$ is $\mathcal{C}^{\infty}$-smooth. Let $Q^{L}=$ $Q \cap\{\operatorname{Re}(z)<0\}$ and $Q^{R}=Q \cap\{\operatorname{Re}(z)>0\}$. We define $\triangle^{L / R}$ similarly.

LEMma 3.1. There exists a $\mathcal{C}^{\infty}$-smooth homemorphism $f: \bar{\triangle} \rightarrow \bar{Q}$, conformal on $\triangle$, such that:

(1) $f(A)=Q^{L}$;

(2) $f(B)=Q^{R}$;

(3) $f(-1)=-1, f(1)=1, f(\sqrt{2}-1)=0, f\left(z_{1}\right)=i$, and $f\left(z_{2}\right)=-i$; and

(4) $f(\bar{z})=\overline{f(z)}$ for all $z \in \triangle$.

Proof. The map $f$ will be the composition of two maps. First, the map

$$
\varphi(z)=\frac{z-(\sqrt{2}-1)}{1-(\sqrt{2}-1) z}
$$

is a holomorphic automorphism of $\triangle$ that maps $B$ to $\triangle^{R}$ with $\varphi(\sqrt{2}-1)=0$, $\varphi\left(z_{1}\right)=i, \varphi\left(z_{2}\right)=-i, \varphi(-1)=-1$, and $\varphi(1)=1$. To see this, let $\tilde{\varphi}(z):=\frac{z+1}{1-z}$, a Möbius transformation that sends $\Delta$ onto the right half-plane with $\tilde{\varphi}(-1)=0$ and $\tilde{\varphi}(1)=\infty$. It sends the piece of $\partial \triangle$ with strictly positive imaginary part to the positive imaginary axis. The map $\tilde{\varphi}(z-\sqrt{2})$ has the corresponding properties for $\triangle(\sqrt{2})$, and $\tilde{\varphi}(z-\sqrt{2})=1 /(\sqrt{2}+1) \cdot \varphi(z)$. It is well known that $\varphi \in$ Aut $_{\text {hol }}(\triangle)$, and it follows that $\varphi$ maps $B$ onto $\triangle^{R}$. Since the piece of $\partial B \cap \triangle$ with positive imaginary part is sent to the positive imaginary axis, we have $\phi\left(z_{1}\right)=i$ and consequently $\varphi\left(z_{2}\right)=-i$. Clearly $\varphi(\bar{z})=\overline{\varphi(z)}$ for all $z \in \triangle$.

To create the second map, let $\psi$ be a conformal map that maps the part of $\triangle^{L}$ with positive imaginary part onto the part of $Q^{L}$ with positive imaginary part. The existence of $\psi$ follows from the Riemann mapping theorem, and-since it is possible to interpolate over three points in $\partial \Delta$ by elements of $\operatorname{Aut}_{\text {hol }}(\triangle)$ - we may assume that $\psi(-1)=-1, \psi(0)=0$, and $\psi(i)=i$. By the Schwarz reflection principle, $\psi$ extends to a map $\psi: \triangle \rightarrow Q$. Then $\psi(\bar{z})=\overline{\psi(z)}$ for all $z \in \triangle$ and so $f:=\psi \circ \varphi$ satisfies (1)-(4). See [3] for the fact that $f$ is $\mathcal{C}^{\infty}$-smooth up to the boundary.

Pick two points $y_{1}^{0} \in \partial B \cap \triangle(\sqrt{2})$ and $y_{2}^{0}=\bar{y}_{1}^{0}$ such that $\operatorname{Re}\left(f\left(y_{i}^{0}\right)\right)=1$ for $i=$ 1,2 (see Figure 3.1). Let $x_{1}^{0}:=y_{1}^{0}-\sqrt{2}$ and $x_{2}^{0}:=y_{2}^{0}-\sqrt{2}$. Since $x_{1}^{0}, x_{2}^{0} \in A$ it follows that $\operatorname{Re}\left(f\left(x_{i}^{0}\right)\right)=\alpha$ for some $-1<\alpha<0$.

For any $0<\delta \leq \frac{1}{2}$, let $I_{0}^{1}(\delta)$ denote the line segment $I_{0}^{1}(\delta):=\{-1\} \times[-\delta, \delta]$ and let $I_{0}^{2}(\delta):=\{0\} \times[-\delta, \delta]$. Let $\gamma_{0}^{1}(\delta):=f^{-1}\left(I_{0}^{1}(\delta)\right)$ and $\gamma_{0}^{2}(\delta):=f^{-1}\left(I_{0}^{2}(\delta)\right)$. Pick a $\delta^{0} \leq \frac{1}{2}$ and choose a smoothly bounded domain $D_{0} \subset \triangle$ as in the figure. The domain $D_{0}$ should have the following properties: 


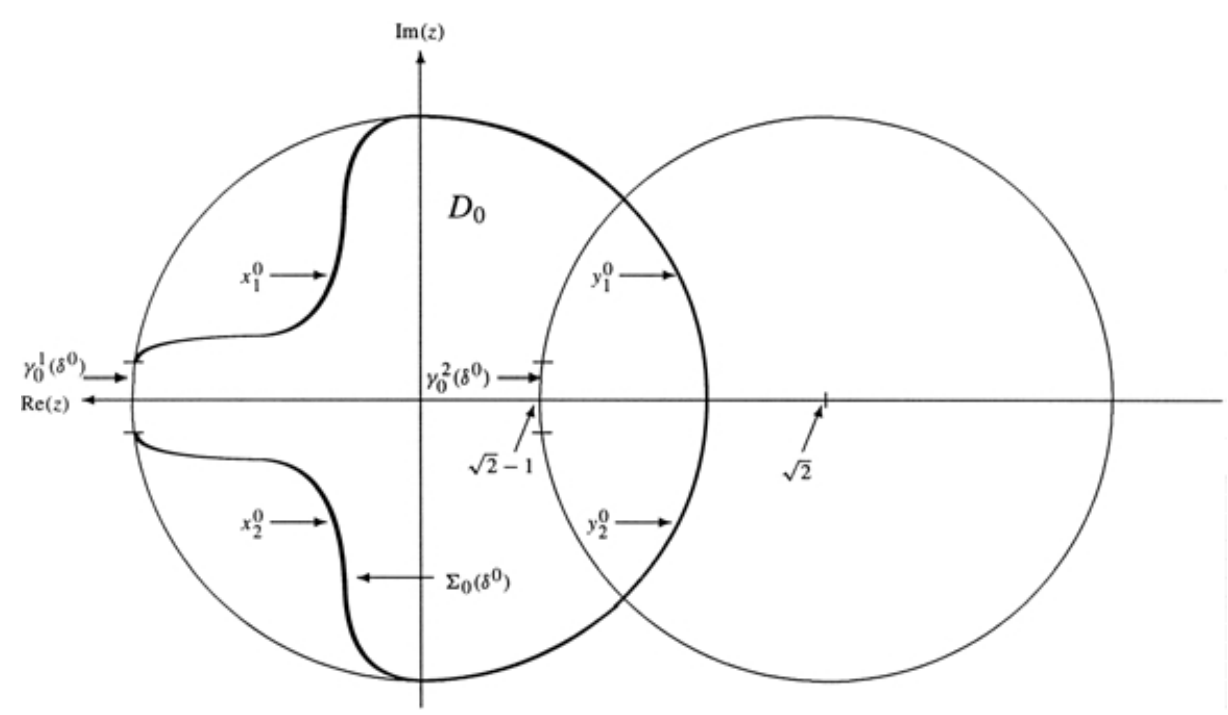

Figure 3.1

(i) $D_{0} \subset \triangle$, and $D_{0}$ agrees with $\triangle$ on the right half-plane;

(ii) $\left[\overline{D_{0}}+\sqrt{2}\right] \cap \overline{D_{0}}=\left\{y_{1}^{0}, y_{2}^{0}\right\}$;

(iii) $\gamma_{0}^{1}\left(\delta^{0}\right) \subset \partial D_{0}$; and

(iv) $\gamma_{0}^{2}\left(\delta^{0}\right) \subset D_{0}$.

Define disks $D_{j}=D_{0}+j \cdot \sqrt{2}$ for all integers $j \geq 0$, and let $f_{j}: \overline{D_{j}} \rightarrow$ $\mathbb{C}$ be defined inductively by $f_{0}:=f$; then $f_{j}(z):=f_{j-1}(z-\sqrt{2})+1$. Let $\left\{x_{1}^{j}, x_{2}^{j}, y_{1}^{j}, y_{2}^{j}\right\}=\left\{x_{1}^{0}, x_{2}^{0}, y_{1}^{0}, y_{2}^{0}\right\}+j \cdot \sqrt{2}$, and for all $\delta \leq \delta^{0}$ let $\gamma_{j}^{i}(\delta)=$ $\gamma_{0}^{i}(\delta)+j \cdot \sqrt{2}, I_{j}^{i}(\delta)=I_{0}^{i}(\delta)+j \cdot \sqrt{2}$, and $\Sigma_{j}(\delta):=\overline{\partial D_{j} \backslash \gamma_{j}^{1}(\delta)}$.

The following properties are easily confirmed for all $j$ and all $\delta \leq \delta^{0}$ :

(v) $\operatorname{Re}\left(f_{j}(z)\right)=-1+j$ for all $z \in \gamma_{j}^{1}(\delta)$;

(vi) $\operatorname{Re}\left(f_{j}(z)\right)=j$ for all $z \in \gamma_{j}^{2}(\delta)$;

(vii) $\operatorname{Re}\left(f_{j}\left(x_{i}^{j}\right)\right)=\alpha+j$, where $-1<\alpha<0$;

(viii) $\operatorname{Re}\left(f_{j}\left(y_{i}^{j}\right)\right)=1+j$;

(ix) $\partial D_{j} \cap \partial D_{j+1}=\left\{y_{1}^{j}, y_{2}^{j}\right\}=\left\{x_{1}^{j+1}, x_{2}^{j+1}\right\}$;

(x) $\partial D_{j} \cap \partial D_{k}=\emptyset$ for $k \neq j \pm 1$; and

(xi) $f_{j}(j \cdot \sqrt{2}+(\sqrt{2}-1))=j=f_{j+1}(j \cdot \sqrt{2}+(\sqrt{2}-1))$.

For $0 \leq \delta \leq \delta^{0}$ and $\varepsilon>0$ we define the following sets:

(xii) $M_{j}(\delta, \varepsilon):=\left\{(z, w) \in \mathbb{C}^{2} \mid z \in \Sigma_{j}(\delta), w=f_{j}(z)+t \cdot i, t \in[-\varepsilon, \varepsilon]\right\}$;

(xiii) $E_{j}(\delta, \varepsilon):=\left\{(z, w) \in \mathbb{C}^{2} \mid z \in \gamma_{j}^{1}(\delta), w=f_{j}(z)+t \cdot i, t \in[-\varepsilon, \varepsilon]\right\}$;

(xiv) $A_{j}^{t}:=\left\{(z, w) \in \mathbb{C}^{2} \mid z \in D_{j}, w=f_{j}(z)+t \cdot i\right\}$; and

(xv) $A_{j}(\varepsilon)=\bigcup_{t \in[-\varepsilon, \varepsilon]} A_{j}^{t}$. 
The sets $M_{j}(\delta, \varepsilon)$ are totally real disks, and the union $M_{j}(\delta, \varepsilon) \cup E_{j}(\delta, \varepsilon)$ bounds the family of analytic disks $A_{j}(\varepsilon)$. Moreover, if $j \neq k$ then $M_{j}(\delta, \varepsilon) \cap M_{k}\left(\delta^{\prime}, \varepsilon^{\prime}\right)=$ $\emptyset$ for all $\delta, \delta^{\prime} \leq \delta^{0}$ and all $\varepsilon, \varepsilon^{\prime}>0$. Hence $\Sigma_{j}(\delta) \cap \Sigma_{j+1}\left(\delta^{\prime}\right)=\left\{y_{1}^{j}, y_{2}^{j}\right\}=$ $\left\{x_{1}^{j+1}, x_{2}^{j+1}\right\}$, so in this case (xii) follows from (vii) and (viii). If $k \neq j \pm 1$ then $\Sigma_{j}(\delta) \cap \Sigma_{k}\left(\delta^{\prime}\right)=\varnothing$ by $(\mathrm{x})$.

For $j \geq 0$ let $q_{j} \in \mathbb{C}^{2}$ denote the point $q_{j}=(j \cdot \sqrt{2}, f(0)+j)$. Then $q_{j} \in A_{j}^{0}$ for all $j$. For a positive number $\mu_{j}$ we will now let $B_{\mu_{j}}\left(q_{j}\right)$ denote the ball of radius $\mu_{j}$ centered at the point $q_{j}$.

LEMMA 3.2. There are sequences of strictly positive real numbers $\delta_{j}^{\prime}<\delta_{j} \leq \delta^{0}$, $\varepsilon_{j}<\varepsilon_{j}^{\prime}$, and $\mu_{j}<\sqrt{2}-1$ such that:

(1) $E_{j+1}\left(\delta_{j+1}, \varepsilon_{j+1}\right) \subset A_{j}\left(\varepsilon_{j}\right)$ for $j=0,1,2, \ldots$; and

(2) any finite union $\bigcup_{j=0}^{m}\left(M_{j}\left(\delta_{j}^{\prime}, \varepsilon_{j}^{\prime}\right) \cup \overline{B_{\mu_{j}}\left(q_{j}\right)}\right)$ is polynomially convex.

Proof. The proof proceeds by induction on $j$. Let $\delta_{0}:=\delta^{0}$, choose any $\varepsilon_{0}>0$, and let $\delta_{0}^{\prime}<\delta_{0}$ and $\varepsilon_{0}^{\prime}>\varepsilon_{0}$. We have that $M_{0}\left(\varepsilon_{0}^{\prime}, \delta_{0}^{\prime}\right)$ is polynomially convex because it projects onto a polynomially convex smooth arc in the plane and the fibers are straight lines. If $\mu_{0}$ is chosen small enough, then (2) holds with $m=0$ and (1) is void at this step.

Assume now that we have chosen $\varepsilon_{j}, \varepsilon_{j}^{\prime}, \delta_{j}, \delta_{j}^{\prime}$, and $\mu_{j}$ for $0 \leq j \leq N$ and that (1) and (2) hold for $0 \leq j, m \leq N$. If $t_{1}$ is small enough, then from (vi), (xi), and the definition of $A_{N}\left(\varepsilon_{N}\right)$ it follows that

$$
\gamma_{N}^{2}\left(t_{1}\right) \times I_{N}^{2}\left(t_{1}\right) \subset A_{N}\left(\varepsilon_{N}\right) .
$$

If $t_{1}^{\prime}<t_{1}$ and if $s_{1}$ and $s_{2}$ are small enough, it follows from (v), (xi), and the definition of $E_{N+1}\left(\varepsilon_{N+1}, \delta_{N+1}\right)$ that

$$
E_{N+1}\left(\varepsilon_{N+1}, \delta_{N+1}\right) \subset \gamma_{N+1}^{1}\left(t_{1}^{\prime}\right) \times I_{N+1}^{1}\left(t_{1}\right) \subset \gamma_{N}^{2}\left(t_{1}\right) \times I_{N}^{2}\left(t_{1}\right)
$$

for all $\varepsilon_{N+1} \leq s_{1}$ and $\delta_{N+1} \leq s_{2}$. Choose $\delta_{N+1}^{\prime}<s_{2}$. If $\varepsilon_{N+1}^{\prime}<s_{1}$ is chosen small enough then $\bigcup_{j=0}^{N}\left(M_{j}\left(\varepsilon_{j}^{\prime}, \delta_{j}^{\prime}\right) \cup \overline{B_{\mu_{j}}\left(q_{j}\right)}\right) \cup M_{N+1}\left(\varepsilon_{N+1}^{\prime}, \delta_{N+1}^{\prime}\right)$ is polynomially convex: By assumption, $K:=\bigcup_{j=0}^{N}\left(M_{j}\left(\varepsilon_{j}^{\prime}, \delta_{j}^{\prime}\right) \cup \overline{B_{\mu_{j}}\left(q_{j}\right)}\right)$ is polynomially convex, and we have that $K \cup M_{N+1}\left(0, \delta_{N+1}^{\prime}\right)$ is polynomially convex since $C:=$ $M_{N+1}\left(0, \delta_{N+1}^{\prime}\right)$ is a simply connected smooth curve and since $K \cap C=\emptyset$ (see e.g. the main theorem in [13] or [14, Thm. 3.1.1]). Let $U, V$ be open sets such that $U \cap V=\emptyset$, and let $K \subset U$ and $C \subset V$. There exists a neighborhood $\Omega$ of $K \cup C$ such that $\Omega \subset \subset U \cup V$ and such that $\bar{\Omega}$ is polynomially convex. If $\varepsilon_{N+1}^{\prime}$ is small enough then $M_{N+1}\left(\varepsilon_{N+1}^{\prime}, \delta_{N+1}^{\prime}\right) \subset \Omega$, and since $M_{N+1}\left(\varepsilon_{N+1}^{\prime}, \delta_{N+1}^{\prime}\right)$ is polynomially convex (it projects onto a smooth arc) it follows that the union with $K$ is polynomially convex (this follows from the Oka-Weil theorem).

Choose $\varepsilon_{N+1}<\varepsilon_{N+1}^{\prime}$ and $\delta_{N+1}^{\prime}<\delta_{N+1}<s_{2}$. Finally, choose $\mu_{N+1}$ small enough.

At this point we fix sequences of numbers as in the previous lemma, and we refer to our sets simply as $M_{j}, M_{j}^{\prime}, E_{j}, A_{j}$, and $B_{j}:=B_{\mu_{j}}\left(q_{j}\right)$. (Here $M_{j}^{\prime}$ denotes the set $M_{j}\left(\delta_{j}^{\prime}, \varepsilon_{j}^{\prime}\right)$.) We let $\tilde{M}$ denote the union $\tilde{M}=\bigcup_{i=0}^{\infty} M_{i} \cup q_{i}$. 


\section{LeMma 3.3. Uniform approximation is not possible on $\tilde{M}$.}

Proof. Define $g\left(q_{j}\right)=j$ and $\left.g\right|_{M_{j}} \equiv 0$ for all $j \geq 0$. Assume to get a contradiction that there is an $h \in \mathcal{O}\left(\mathbb{C}^{2}\right)$ such that $\sup _{x \in \tilde{M}}\{|h(x)-g(x)|\}<\frac{1}{2}$.

Let $N \geq 1$ be a natural number such that $\|h\|_{E_{0}} \leq N$. By assumption we have that $\left|h\left(q_{N+1}\right)\right|>N$. By the maximum principle, $h \mid \frac{}{A_{N+1}^{0}}$ must take its maximum on $\partial A_{N+1}^{0} \subset M_{N+1} \cup E_{N+1}$. By assumption $\|h\|_{M_{N+1}}<\frac{1}{2}$, so there exists a point $p_{N} \in E_{N+1} \subset A_{N}$ (by Lemma 3.2(1)) such that $\left|h\left(p_{N}\right)\right|>N$. Then $p_{N}$ lies in $A_{N}^{t_{N}}$ for some $t_{N} \in\left[-\varepsilon_{N}, \varepsilon_{N}\right]$.

Again $h \mid \frac{}{A_{N}^{t_{N}}}$ must take its maximum on $\partial A_{N}^{t_{N}} \subset M_{N} \cup E_{N}$, and this must happen at a point $p_{N-1} \in E_{N} \subset A_{N-1}$. Continuing this argument, we end up with a point $p_{0} \in E_{0}$ such that $\left|h\left(p_{0}\right)\right|>N$, which is a contradiction.

Now we regard the set $\tilde{M}$ as being contained in $\mathbb{C}^{2} \times\{0\} \subset \mathbb{C}^{2} \times \mathbb{C}=\mathbb{C}^{3}$.

LEMma 3.4. There exists a $\mathcal{C}^{\infty}$-smooth and proper embedding $\phi: \mathbb{R}^{2} \rightarrow \mathbb{C}^{3}$ such that $\tilde{M} \subset M:=\phi\left(\mathbb{R}^{2}\right)$ and such that $M$ is totally real and polynomially convex.

Proof. We start by choosing a $\mathcal{C}^{\infty}$-smooth proper immersion $g: \mathbb{R} \rightarrow \mathbb{C}$ such that $g(\mathbb{R})$ contains all the curves $\Sigma_{j}^{\prime}$. The image $g(\mathbb{R})$ should look like the curve in Figure 3.2.

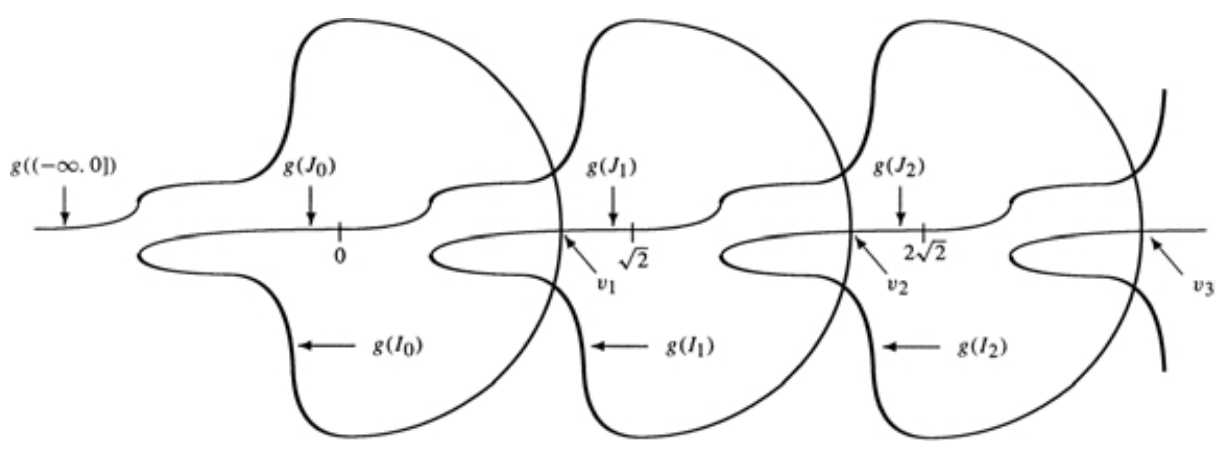

Figure 3.2

For each $j$ we first connect $\Sigma_{j}^{\prime}$ to $\Sigma_{j+1}^{\prime}$ by a smooth curve $l_{j}$ between the endpoint of $\Sigma_{j}^{\prime}$ with negative imaginary part and the endpoint of $\Sigma_{j+1}^{\prime}$ with positive imaginary part. The curve $l_{j}$ will intersect $\Sigma_{j-1}^{\prime}$ at a single point $v_{j}$ for $j \geq 1$. Let $l_{-1}$ be a smooth curve between the point $\{-1\}$ and the endpoint of $\Sigma_{0}^{\prime}$ with positive imaginary part. Let $\Gamma$ denote the union of all these curves with the interval $(-\infty,-1)$. The image $g(\mathbb{R})$ should be a smoothing of $\Gamma$ that creates no new self-intersection points; $g(\mathbb{R})$ should contain the curves $\Sigma_{j}^{\prime}$, and we make sure that it passes through all points $\{j \cdot \sqrt{2}\}$ for $j \geq 0$. Moreover we make sure that, for 
all real numbers $t$, there are only a finite number of points on $g(\mathbb{R})$ whose real part equals $t$. If we let $I_{k}:=[2 k, 2 k+1]$ and $J_{k}:=[2 k+1,2 k+2]$, then we may assume the following:

(a) $\left.g\right|_{I_{k}},\left.g\right|_{J_{k}}$, and $\left.g\right|_{\{t \leq 0\}}$ are all embeddings;

(b) $g\left(I_{k}\right)=\Sigma_{k}^{\prime}$;

(c) $g\left(2 k+\frac{4}{3}\right)=v_{k}$ for $k \geq 1$;

(d) $g\left(2 k+\frac{5}{3}\right)=k \cdot \sqrt{2}$ for $k \geq 0$;

(e) $g\left(I_{j}\right) \cap g\left(I_{k}\right)=\left\{y_{1}^{j}, y_{2}^{j}\right\}$ if $k=j+1$, and if $k \neq j \pm 1$ then the intersection is empty;

(f) $g\left(I_{j}\right) \cap g\left(J_{k}\right)=\left\{v_{k}\right\}$ if $j=k-1 \geq 0$, and otherwise the intersection is empty;

(g) $g\left(J_{i}\right) \cap g\left(J_{k}\right)=\emptyset$ for all $i \neq k$; and

(h) $g(\{t<0\}) \cap g(\{t>0\})=\emptyset$.

Choose a $\mathcal{C}^{\infty}$-smooth function $h: \mathbb{R} \rightarrow \mathbb{C}$ such that the following hold:

(i) $h(t)=f_{j}(g(t))$ for all $t \in I_{j}$;

(j) $\operatorname{Re}\left(h\left(2 j+\frac{4}{3}\right)\right) \neq \operatorname{Re}\left(f_{j-1}\left(v_{j}\right)\right)$; and

(k) $h\left(2 j+\frac{5}{3}\right)=f(0)+j$.

We define first a smooth, proper embedding $\psi: \mathbb{R}^{2} \rightarrow \mathbb{C}^{2}$ by

$$
\psi(x, y):=(g(x), h(x)+y \cdot i) .
$$

This map is injective because our choices have been made such that if $g\left(x_{1}\right)=$ $g\left(x_{2}\right)$ then $\operatorname{Re}\left(h\left(x_{1}\right)\right) \neq \operatorname{Re}\left(h\left(x_{2}\right)\right)$ if $x_{1} \neq x_{2}$.

It is clear that $\psi\left(\mathbb{R}^{2}\right)$ is totally real. Since $\tilde{M} \subset \psi\left(\mathbb{R}^{2}\right)$, it follows from Lemma 3.3 that uniform approximation is not possible on $\psi\left(\mathbb{R}^{2}\right)$. To get a polynomially convex surface we will now bend "most" of $\psi\left(\mathbb{R}^{2}\right)$ into $\mathbb{C}^{3}$, keeping $\tilde{M}$ in $\mathbb{C}^{2}$.

Let $P_{j}^{\prime} \subset \mathbb{R}^{2}$ denote the set $P_{j}^{\prime}:=I_{j} \times\left[-\varepsilon_{j}^{\prime}, \varepsilon_{j}^{\prime}\right]$. We have:

(1) $\psi\left(P_{j}^{\prime}\right)=M_{j}^{\prime}$ for all $j \geq 0$; and

(m) $\psi\left(\left(2 j+\frac{5}{3}, 0\right)\right)=q_{j}$ for all $j \geq 0$.

Let $P_{j}:=\psi^{-1}\left(M_{j}\right) \subset \subset P_{j}^{\prime}$. For each $j$ let $S_{j} \subset \mathbb{R}^{2}$ be a closed disk centered at $\left(2 j+\frac{5}{3}, 0\right)$ such that $\psi\left(S_{j}\right) \subset \subset B_{j}$. Let $\chi: \mathbb{R}^{2} \rightarrow[0,1]$ be a $\mathcal{C}^{\infty}$-smooth function such that

(n) $\left.\chi\right|_{P_{j} \cup\{(2 j+5 / 3,0)\}} \equiv 0$ and

(o) $\chi(x, y)=1$ for all $(x, y) \in \mathbb{R}^{2} \backslash\left(\bigcup_{i=0}^{\infty}\left(P_{i}^{\prime} \cup S_{i}\right)\right)$.

We define our final embedding by

$$
\phi:=\psi+\chi \cdot \operatorname{Re}(g) \cdot \mathrm{e}_{3} .
$$

Then $\phi\left(\mathbb{R}^{2}\right)$ is polynomially convex. Note first that $Z^{\prime}:=\phi\left(\bigcup_{i=0}^{m}\left(P_{i}^{\prime} \cup S_{i}\right)\right)$ is polynomially convex for all $m \in \mathbb{N}$. It follows from Lemma 3.2 that $Z:=$ $\psi\left(\bigcup_{i=0}^{m}\left(P_{i}^{\prime} \cup S_{i}\right)\right) \subset \mathbb{C}^{2}$ is polynomially convex, and it follows from [14, Thm. 6.3.1] together with the Oka-Weil theorem that $\mathcal{P}(Z)=\mathcal{C}(Z)$. Since $Z^{\prime}$ is a graph 
over $Z \subset \mathbb{C}^{2}$ in $\mathbb{C}^{3}$ it follows that $\mathcal{P}\left(Z^{\prime}\right)=\mathcal{C}\left(Z^{\prime}\right)$, and $Z^{\prime}$ is polynomially convex by $[14$, Thm. 1.2.10].

Let $\pi_{3}$ denote the projection onto the third coordinate in $\mathbb{C}^{3}$. Restricted to $M$ we have that $\pi_{3}$ is a real-valued function. According to [14, Thm. 1.2.16], a compact set $K \subset M$ is polynomially convex if and only if $\pi_{3}^{-1}\left(t_{0}\right) \cap K$ is polynomially convex for each $t_{0} \in \mathbb{R}$. Let $X_{j}$ be a compact exhaustion of $\mathbb{R}^{2}$ by squares that pick up exactly one pair $\left(P_{j}^{\prime}, S_{j}\right)$ for each increase of $j$. The following shows that $\phi\left(X_{j}\right)$ is polynomially convex for each $j$. Consider first a set $Y:=$ $\left(\bigcup_{i=1}^{k}\left\{x_{i}\right\} \times[-R, R]\right) \cup\left(\bigcup_{i=0}^{m} P_{i}^{\prime} \cup S_{i}\right)$, where $x_{1}, \ldots, x_{k}$ is a finite set of points and where $R \in \mathbb{R}^{+}$and $m \in \mathbb{N}^{+}$. We have seen that $\phi\left(\bigcup_{i=0}^{m} P_{i}^{\prime} \cup S_{i}\right)$ is polynomially convex, so by [13] the set $\phi(Y)$ is polynomially convex. It now follows from [14, Thm. 6.3.1] and [13] that $\mathcal{P}(\phi(Y))=\mathcal{C}(\phi(Y))$; hence any relatively closed subset of $\phi(Y)$ is polynomially convex.

Fix a $t_{0}$ and assume that $\pi_{3}^{-1}\left(t_{0}\right) \cap \phi\left(X_{j}\right) \neq \emptyset$. Define

$$
L:=\phi^{-1}\left(\pi_{3}^{-1}\left(t_{0}\right) \cap \phi\left(X_{j}\right)\right)=\left\{(x, y) \in X_{j} \mid \chi(x, y) \cdot \operatorname{Re}(g(x))=t_{0}\right\} .
$$

By assumption there are only a finite number of $x_{j}$ such that $\operatorname{Re}\left(g\left(x_{j}\right)\right)=t_{0}$. Since $\chi \equiv 1$ on $L \backslash\left(\bigcup_{i=0}^{j} P_{i}^{\prime} \cup S_{i}\right)$, it follows that $L \backslash\left(\bigcup_{i=0}^{j} P_{i}^{\prime} \cup S_{i}\right) \subset \bigcup_{j=1}^{k}\left\{x_{j}\right\} \times$ $[-R, R]$ for some $R \in \mathbb{R}$. So $L$ is a closed subset of a set on the same form as $Y$, and by the preceding arguments $\phi(L)$ is polynomially convex.

Adding a third component to the embedding $\psi$ does not change the fact that the image is totally real.

\section{References}

[1] H. Alexander, A Carleman theorem for curves in $\mathbb{C}^{n}$, Math. Scand. 45 (1979), 70-76.

[2] H. Alexander and J. Wermer, Several complex variables and Banach algebras, Grad. Texts in Math., 35, Springer-Verlag, New York, 1998.

[3] S. R. Bell, The Cauchy transform, potential theory, and conformal mapping, Stud. Adv. Math., CRC Press, Boca Raton, FL, 1992.

[4] T. Carleman, Sur un theoreme de Weierstrass, Arkiv för Matematik, Astronomi och Fysik 20B (1927).

[5] P. M. Gauthier and E. S. Zeron, Approximation on arcs and dendrites going to infinity in $\mathbb{C}^{n}$, Canad. Math. Bull. 45 (2002), 80-85.

[6] L. Hoischen, Eine Verschäfung eines approximationssatzes von Carleman, J. Approx. Theory 9 (1973), 272-277.

[7] L. Hörmander and J. Wermer, Uniform approximation on compact sets in $\mathbb{C}^{n}$, Math. Scand. 23 (1968), 5-21.

[8] P. Manne, Carleman approximation on totally real submanifolds of a complex manifold, Several complex variables (Stockholm, 1987/1988), Math. Notes, 38, pp. 519-528, Princeton Univ. Press, Princeton, NJ, 1993.

[9] - Carleman approximation by entire functions on the union of two totally real subspaces of $\mathbb{C}^{n}$, Canad. Math. Bull. 37 (1994), 522-526.

[10] J. Nunemacher, Approximation theory on totally real submanifolds, Math. Ann. 224 (1976), 129-141.

[11] M. Range and Y. T. Siu, $\mathcal{C}^{k}$ approximation by holomorphic functions and $\bar{\partial}$-closed forms on $\mathcal{C}^{k}$ submanifolds of a complex manifold, Math. Ann. 210 (1974), 105-122. 
[12] S. Scheinberg, Uniform approximation by entire functions, J. Analyse Math. 29 (1976), 16-18.

[13] G. Stolzenberg, Uniform approximation on smooth curves, Acta Math. 115 (1966), 185-198.

[14] E. L. Stout, Polynomial convexity, Progr. Math., 261, Birkhäuser, Boston, 2007.

[15] K. Weierstrass, Über die analytische Darstellbarkeit sogenannter willkürlicher Functionen reeller Argumente, Sitzungberichte der Königl, Preuss. Akademie der Wissenschaften zu Berlin (1885), 633-639, 789-805.

Matematisk Institutt

Universitetet i Oslo

Postboks 1053 Blindern

0316 Oslo

Norway

erlendfw@math.uio.no 